\title{
Power Generating Characteristics of Zinc Oxide Nanorods Grown on a Flexible Substrate by a Hydrothermal Method
}

\begin{abstract}
Jae-hoon Choi ${ }^{\dagger}$, Xueqiu You*, Chul Kim**, Jungil Park* and James Jungho Pak***
Abstract - This paper describes the power generating property of hydrothermally grown $\mathrm{ZnO}$ nanorods on a flexible polyethersulfone (PES) substrate. The piezoelectric currents generated by the $\mathrm{ZnO}$ nanorods were measured when bending the $\mathrm{ZnO}$ nanorod by using I-AFM, and the measured piezoelectric currents ranged from 60 to $100 \mathrm{pA}$. When the PtIr coated tip bends a $\mathrm{ZnO}$ nanorod, piezoelectrical asymmetric potential is created on the nanorod surface. The Schottky barrier at the $\mathrm{ZnO}$-metal interface accumulates elecntrons and then release very quickly generating the currents when the tip moves from tensile to compressed part of $\mathrm{ZnO}$ nanorod. These $\mathrm{ZnO}$ nanorods were grown almost vertically with the length of $300-500 \mathrm{~nm}$ and the diameter of $30-60 \mathrm{~nm}$ on the $\mathrm{Ag} / \mathrm{Ti} / \mathrm{PES}$ substrate at $90^{\circ} \mathrm{C}$ for 6 hours by hydrothermal method. The metal-semiconductor interface property was evaluated by using a HP 4145B Semiconductor Parameter Analyzer and the piezoelectric effect of the $\mathrm{ZnO}$ nanorods were evaluated by using an I-AFM. From the measured $I-V$ characteristics, it was observed that $\mathrm{ZnO}-\mathrm{Ag}$ and $\mathrm{ZnO}-\mathrm{Au}$ metal-semiconductor interfaces showed an ohmic and a Schottky contact characteristics, respectively. ANSYS finite element simulation was performed in order to understand the power generation mechanism of the $\mathrm{ZnO}$ nanorods under applied external stress theoretically.
\end{abstract}

Keywords: Hydrothermal method, I-AFM, Piezoelectric effect, Schottky contact, Zinc oxide

\section{Introduction}

Nanoscale semiconductor materials have attracted great interests of researchers because of their importance not only in fundamental research areas but also in practical applications. Among many nanoscale semiconductor materials, $\mathrm{ZnO}$ is a typical piezoelectric inorganic semiconducting material, which has a wide direct band gap of $3.37 \mathrm{eV}$, a large excitation binding energy of $60 \mathrm{meV}$, piezoelectricity, excellent chemical and thermal stability, and biocompatibility [1], [2]. In order to grow $\mathrm{ZnO}$ nanostructures, various techniques have been developed for the synthesis of the $\mathrm{ZnO}$ nanostructures including vapor-liquid-solid (VLS) method, chemical vapor deposition (CVD), and hydrothermal method [3]-[6]. VLS and CVD methods require sophisticated equipments and strict conditions such as single-crystalline substrates, relatively high process temperature $\left(>500^{\circ} \mathrm{C}\right)$, and vacuum conditions $\left(\sim 10^{-2}\right.$ Torr $)$ [7]. The hydrothermal method is substrate independent, convenient, and low cost for large-scale preparation of well-ordered $\mathrm{ZnO}$ nanorod arrays.

Recently, $\mathrm{ZnO}$ nanostructures have been studied for power generating devices due to piezoelectric and semiconducting properties of $\mathrm{ZnO}$ [8]. The $\mathrm{ZnO}$ nanorods can

$\dagger$ Corresponding Author: School of Electrical Engineering, Korea University, Korea.

* School of Electrical Engineering, Korea University, Korea. (pak@korea.ac.kr)

** Department of Micro/Nano Systems, Korea University, Korea. (pak@korea.ac.kr)

Received: March 23, 2010; Accepted: July 21, 2010 be used in systems such as foldable or reformable power source, artificial skins, touch sensors and flexible displays because it is possible for $\mathrm{ZnO}$ nanostructures to generate the current and potential under an external mechanical strain [9].

This paper describes the power generating properties of hydrothermally grown $\mathrm{ZnO}$ nanorods on a flexible polyethersulfone (PES) substrate. The $\mathrm{ZnO}$ nanorods were grown successfully on the flexible substrate by a hydrothermal method, and then they were evaluated by using a scanning electron microscopy (SEM) and an energy dispersive X-ray analysis (EDX). The power generating properties of the $\mathrm{ZnO}$ nanorods were examined by using a conductive atomic force microscopy (I-AFM) and the HP 4145B Semiconductor Parameter Analyzer. Also, the stress-strain distributions for the $\mathrm{ZnO}$ nanorods were analyzed by using ANSYS finite element analysis software.

\section{Experiments}

\subsection{Materials and Chemicals}

All the chemicals used in the fabrication process are commercially available and they were used without further purification since they are of electronic grade. Sodium hydroxide $(\mathrm{NaOH})$, zinc nitrate hexahydrate, zinc acetate dehydrate, and hexamethylenetriamine (HMTA) were purchased from Sigma-Aldrich. 


\subsection{Growth of ZnO Nanorods on Flexible PES Sub- strate}

Fig. 1 shows the fabrication procedure of growing $\mathrm{ZnO}$ nanorods on a flexible PES substrate. PES film $(4 \mathrm{~cm} \times 4$ $\mathrm{cm}$ and $500 \mu \mathrm{m}$ thick) was used as a starting flexible substrate and a thin Ti layer was deposited on the PES film by e-beam evaporation as an adhesion layer. Ag was subsequently deposited on the Ti/PES substrate by thermal evaporation. $\mathrm{ZnO}$ nanorods on the PES were prepared by a hydrothermal method. A mixture of $30 \mathrm{mM} \mathrm{NaOH}$ solution in methanol and $10 \mathrm{mM}$ zinc acetate dehydrate in methanol was used as a seed solution for the $\mathrm{ZnO}$ nanorods under stirring at $60^{\circ} \mathrm{C}$ for 2 hours. This seed solution was spincoated onto a $\mathrm{Ag} / \mathrm{Ti} / \mathrm{PES}$ substrate at $1000 \mathrm{rpm}$ for $30 \mathrm{~s}$. The uniformity and density of the $\mathrm{ZnO}$ seed layer on the substrate depend on the spin-coating speed and time [10], [11]. Also, the density of $\mathrm{ZnO}$ nanorods depends on the density of $\mathrm{ZnO}$ seed layer [11]. After coating the $\mathrm{ZnO}$ seed layer, the substrate was dried and annealed at $150{ }^{\circ} \mathrm{C}$ for 10 min to enhance the seed layer adhesion to the substrate. $\mathrm{ZnO}$ nanorods were then formed in a growth solution, which was a mixture of $25 \mathrm{mM}$ HMTA and $25 \mathrm{mM}$ zinc nitrate hexahydrate in this experiment at $90^{\circ} \mathrm{C}$ for 6 hours [12]. The substrate was then rinsed with deionized water and dried with $\mathrm{N}_{2}$ gas.

\subsection{Characteristics Evaluation of ZnO Nanorods}

The geometry of the grown $\mathrm{ZnO}$ nanorods was observed by using a field-emission scanning electron microscope (FE-SEM, Hitachi S-4300). The element composition of the $\mathrm{ZnO}$ nanorods was examined by using an energy dispersive X-ray spectroscope (EDX, Horiba EX-200). The contacts between $\mathrm{ZnO}$ nanorods and metal electrodes ( $\mathrm{Ag}$ or $\mathrm{Au}$ ) were evaluated by using HP 4145B semiconductor parameter analyzer.

Both $I-V$ characteristic curves of a $\mathrm{ZnO}$ nanorod and the generating currents from the bending $\mathrm{ZnO}$ nanorods were measured by using the I-AFM (XE-100, PSIA) in contact mode. Fig. 2 shows the schematic illustration of an I-AFM based measurement setup for measuring $I-V$ characteristics and piezoelectric effect of the grown $\mathrm{ZnO}$ nanorods. The bottom of $\mathrm{ZnO}$ nanorods was electrically connected to a metal holder through conductive silver paste. The free ends of the $\mathrm{ZnO}$ nanorods were bent by using a $30 \mathrm{~nm}$ radius IAFM Si tip which is coated with alloyed PtIr. In order to measure the $I-V$ characteristic curves of a single $\mathrm{ZnO}$ nanorod, a PtIr coated Si tip was used in static contact with the top of a single $\mathrm{ZnO}$ nanorod. A voltage was swept from 2.7 to $2.7 \mathrm{~V}$ between the $\mathrm{ZnO}$ nanorod and the I-AFM tip, and then the current was measured by I-AFM. For measuring the current generated by bending $\mathrm{ZnO}$ nanorods, $\mathrm{ZnO}$ nanorods were bent by scanning the I-AFM tip over the $\mathrm{ZnO}$ nanorods. This current was measured between the IAFM tip and the metal holder at zero applied voltage.

In order to understand the piezoelectric characteristic of each $\mathrm{ZnO}$ nanorod theoretically, a three-dimensional (3-D) stress-strain simulation was performed when a $\mathrm{ZnO}$ nanorod was bent by using ANSYS (ANSYS. Inc.), a finite element simulation tool.

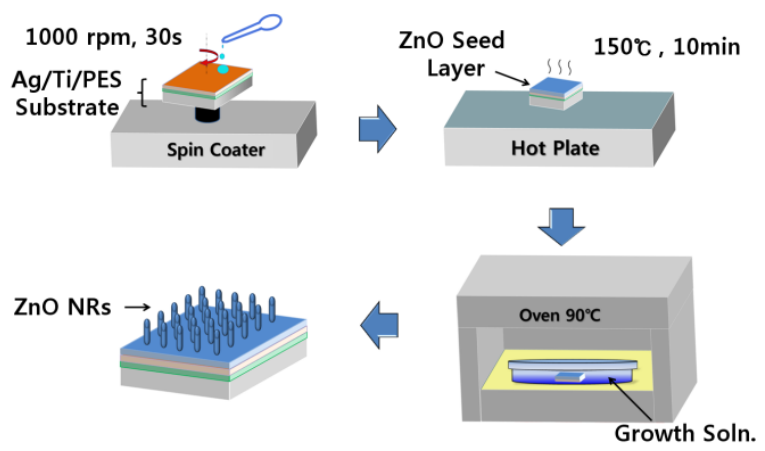

Fig. 1. Schematic illustration: fabrication of $\mathrm{ZnO}$ nanorods on the $\mathrm{Ag} / \mathrm{Ti} / \mathrm{PES}$ substrate by a hydrothermal method.

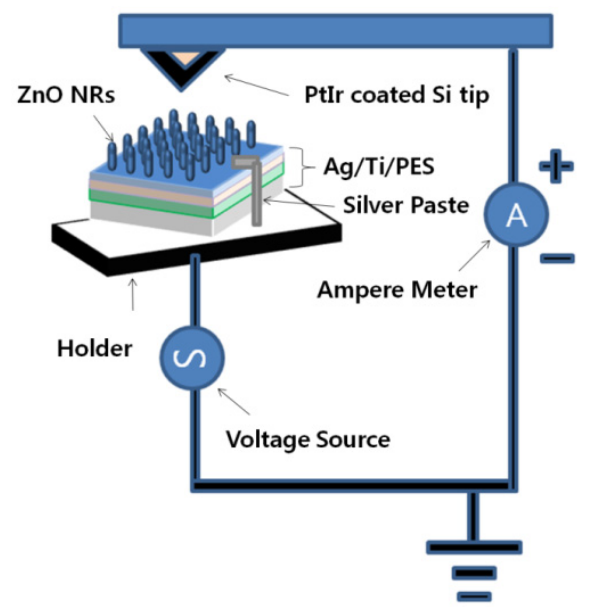

Fig. 2. Schematic of I-AFM based measurement setup for the $I-V$ characteristics and piezoelectric effect of $\mathrm{ZnO}$ nanorods.

\section{Results and Discussion}

Fig. 3 shows the SEM images of the grown $\mathrm{ZnO}$ nanorods on the $\mathrm{Ag} / \mathrm{Ti} / \mathrm{PES}$ substrate. $\mathrm{ZnO}$ nanorods were quite uniformly grown with the length of 300-500 $\mathrm{nm}$ and the diameter 30-50 $\mathrm{nm}$ as shown in Fig. 3(b). The nanorod number density can be as high as five billion rods per $\mathrm{cm}^{2}$. The cross-section SEM image (Fig. 3c) shows that the $\mathrm{ZnO}$ nanorods were grown almost vertically. From the EDX spectrum result in Fig. 4, the atomic ratio of $\mathrm{Zn}$ to $\mathrm{O}$ is calculated to be $1: 1.01$, confirming the purity of $\mathrm{ZnO}$ nanorods.

The linear $I-V$ curve in Fig. 5(a) clearly indicates that $\mathrm{ZnO}-\mathrm{Ag}$ interface formed an ohmic contact. It has been reported that there is no electron energy barrier at the interface because the electron affinity of $\mathrm{ZnO}$ is $4.5 \mathrm{eV}$ and the work function of $\mathrm{Ag}$ is $4.26 \mathrm{eV}$ [9]. While Fig. 5(b) shows a Schottky contact characteristic between the Au electrode and the grown $\mathrm{ZnO}$ nanorods. Because the work function 
of $\mathrm{Au}$ is $5.10 \mathrm{eV}$ [13], which is substantially greater than the electron affinity of $\mathrm{ZnO}$, there is an electron energy barrier at the interface.

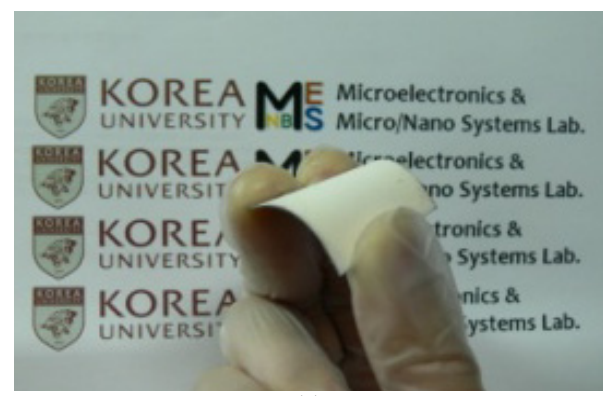

(a)

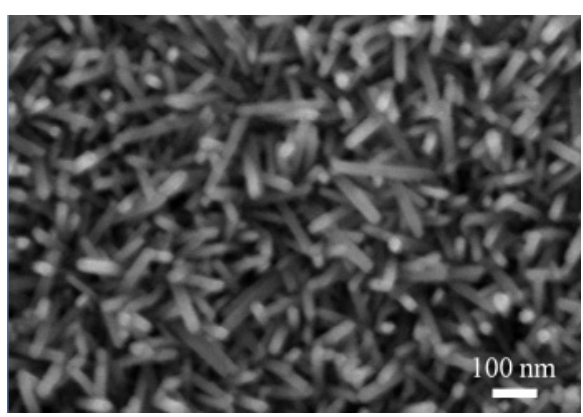

(b)

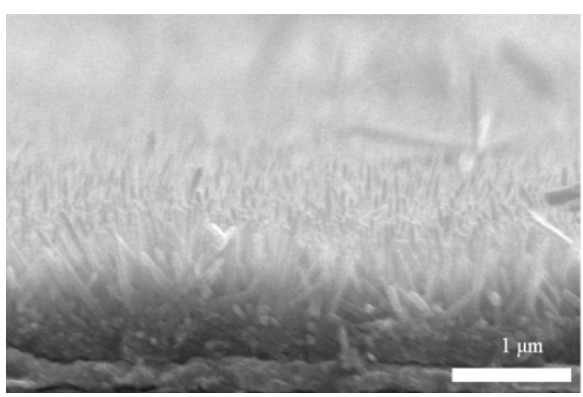

(c)

Fig. 3. $\mathrm{ZnO}$ nanorods on a $\mathrm{Ag} / \mathrm{Ti}$ coated PES substrate. (a) Photographic image of the flexible $\mathrm{ZnO}$ nanorods. SEM images of the $\mathrm{ZnO}$ nanorods uniformly grown on a flexible substrate (b) top view and (c) cross-section view.

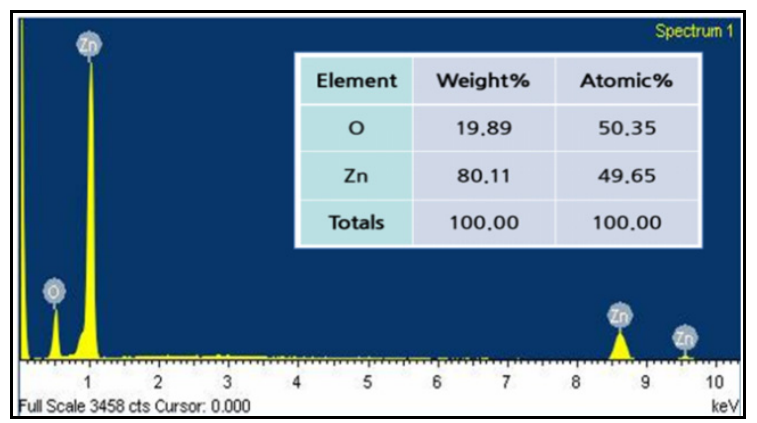

Fig. 4. The EDX spectrum of the grown $\mathrm{ZnO}$ nanorods on a flexible PES substrate. The inserted table shows the weight percentage and atomic percentage of the $\mathrm{ZnO}$ nanorods element composition.

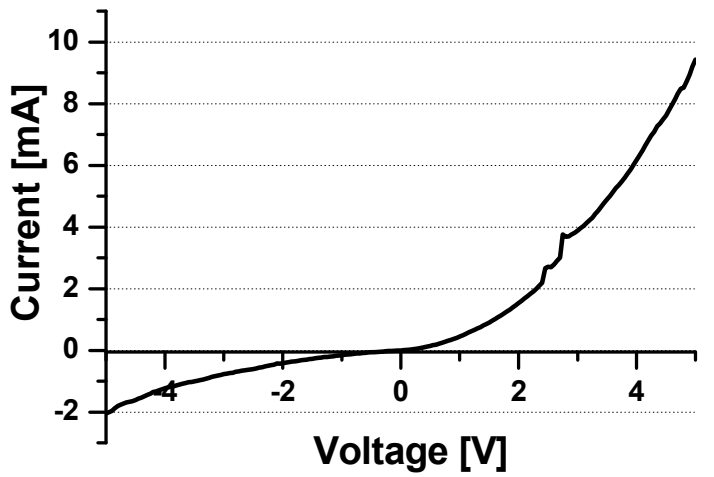

(a)

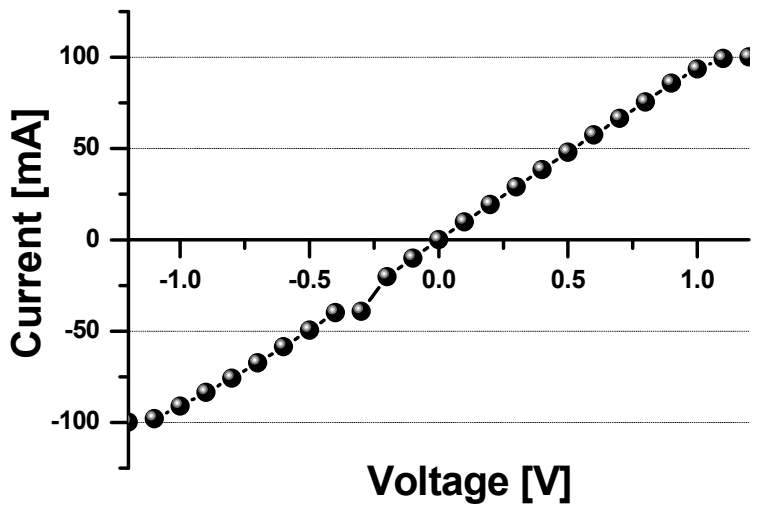

(b)

Fig. 5. $I-V$ curves of (a) $\mathrm{ZnO}$ nanorods- $\mathrm{Ag}$ contact and (b) $\mathrm{ZnO}$ nanorods-Au contact.

As shown in Fig. 6(a), the measured $I-V$ curves between the alloyed PtIr coated I-AFM tip and the grown $\mathrm{ZnO}$ nanorod showed a Schottky contact curve. The negative current means that the currents flow from the grounded end to the tip. When a force of $200 \mathrm{nN}$ was applied by scanning the I-AFM tip (spring constant of $0.2 \mathrm{~N} / \mathrm{m}$ ), 60-100 pA negative currents were measured by using the I-AFM as shown in Fig. 6(b).

The ANSYS simulation results showed the stress-strain distributions when the $\mathrm{ZnO}$ nanorods were bent by using the AFM tip in Fig. 7. The 3-D stress-strain simulation was performed with a $\mathrm{ZnO}$ nanorod of diameter $40 \mathrm{~nm}$ and length $400 \mathrm{~nm}$ which has the elastic modulus (Young's modulus) of about $170 \mathrm{GPa}$ [14], the Poisson's ratio of 0.303 [15]. The $\mathrm{ZnO}$ nanorod was modeled as a solid cylinder shape as shown in Fig. 7(a). The bottom of the $\mathrm{ZnO}$ nanorod was fixed on the surface of substrate. For bending the nanorod, $200 \mathrm{nN}$ force at the top of $\mathrm{ZnO}$ nanorod was applied. Fig. 7(b) shows the negative strain caused by compression and the positive strain caused by expansion. From the piezoelectric effect, the compressed surface has relatively negative potential compared to the tensile surface [16]. The principle of current-output process is accumulating/releasing electrons at/from the interface between the IAFM tip and $\mathrm{ZnO}$ nanorod. When the I-AFM tip bends the $\mathrm{ZnO}$ nanorod, a positive potential is induced on the tensile surface. The electrons from the grounded I-AFM tip move to the PtIr coated tip. Reverse biased Schottky barrier with 
$\mathrm{ZnO}$ nanorods block the electron's moving from the tip to the $\mathrm{ZnO}$ nanorod. As the tip moves to the compressed surface with negative potential, the tip has a forward biased Schottky barrier with the $\mathrm{ZnO}$ nanorod. Because the potential of the I-AFM tip is higher than the potential of compressed $\mathrm{ZnO}$ nanorod, the accumulated electrons on the tip release very quickly and generate the current signals. Therefore 60-100 pA negative currents were measured.

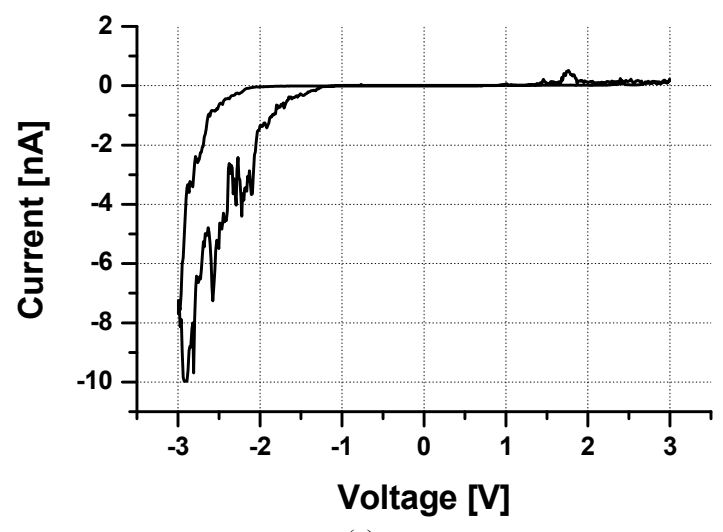

(a)

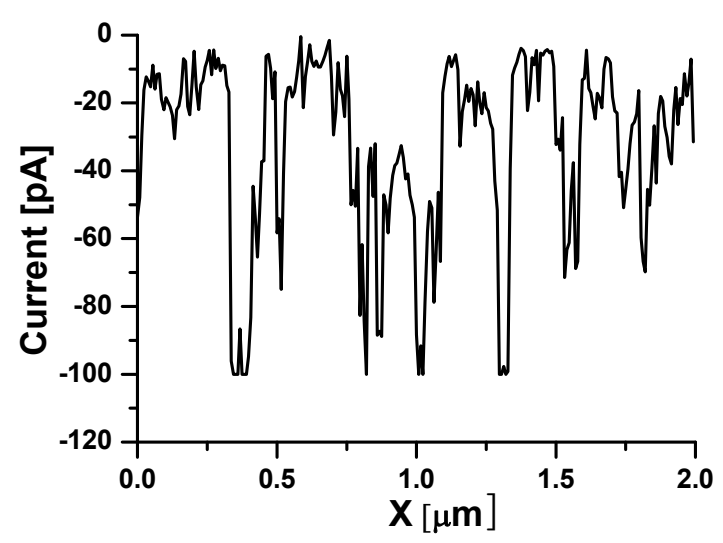

(b)

Fig. 6. (a) $I-V$ curves of $\mathrm{ZnO} / \mathrm{PtIr}$ coated $\mathrm{Si}$ tip, showing a Schottky contact curve. (b) The output current signals generated by $\mathrm{ZnO}$ nanorods when scanned by I-AFM tip.

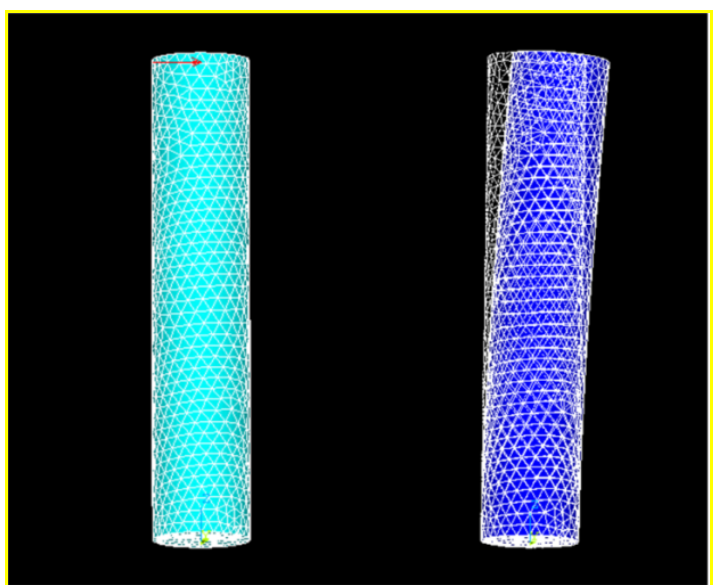

(a)

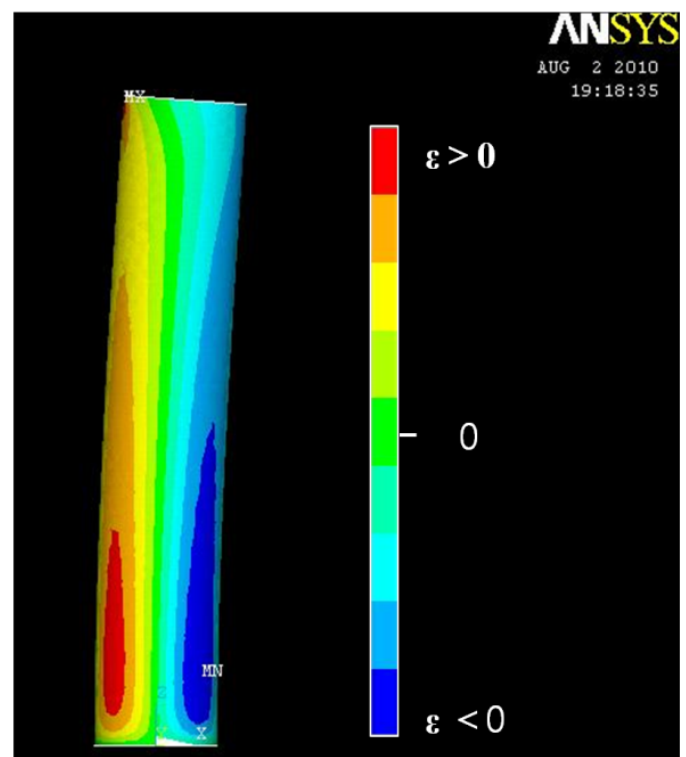

(b)

Fig. 7. The stress-strain distributions of ANSYS simulation. (a) Schematic of $\mathrm{ZnO}$ nanorod bent by $200 \mathrm{nN}$ on top of the nanorod. (b) The calculated strain $\varepsilon$ distribution of deformed $\mathrm{ZnO}$ nanorod.

\section{Conclusion}

$\mathrm{ZnO}$ nanorods were uniformly synthesized on a $\mathrm{Ag} / \mathrm{Ti} / \mathrm{PES}$ flexible substrate by using a simple hydrothermal method. $\mathrm{ZnO}$ nanorods were almost vertically grown with 300-500 nm lengths and 30-50 nm diameters. The ohmic contact formation of $\mathrm{ZnO}-\mathrm{Ag}$ and Schottky contact formation of $\mathrm{ZnO}-\mathrm{Au}$ have been demonstrated by $I-V$ characteristic curves from semiconductor parameter analyzer. 60-100 pA negative currents were obtained from the IAFM tip in contact mode AFM measurements. The power generation based on piezoelectric property of $\mathrm{ZnO}$ nanorods can be explained by stress-strain distribution analysis by finite element method simulation and I-AFM results. The combination of metal-semiconductor interface property and piezoelectric property of $\mathrm{ZnO}$ nanorods realize the power generation

\section{Acknowledgements}

This research was supported by Seoul R\&BD Program (No. 10920) and the National Research Foundation of Korea through a grant provided by Ministry of Education, Science and Technology in 2009(No. K2060100000209E0100-00200). The authors are grateful to Park Systems Corp. for their help in measuring the piezoelectric current from the hydrothermally grown $\mathrm{ZnO}$ nanorods with their IAFM. 


\section{References}

[1] Ü. Özgür, Ya. I. Alivov, C. Liu, A. Teke, M. A. Reshchikov, S. Doğan, V. Avrutin, S. -J. Cho, and H. Morkoç, "A comprehensive review of $\mathrm{ZnO}$ materials and devices", Journal of Applied Physics, Vol.98, p.041301, Aug. 2005.

[2] Michael H. Huang, Samuel Mao, Henning Feick, Haoquan Yan, Yiying Wu, Hannes Kind, Eicke Weber, Richard Russo, and Peidong Yang, "Room-temperature ultraviolet nanowire nanolasers", Science, Vol.292, No.8, pp.1897-1899, June 2001.

[3] Yuko Satoh, Shigeo Ohshio, and Hidetoshi Saitoh, "Photoluminescence spectroscopy of highly oriented $\mathrm{Y}_{2} \mathrm{O}_{3}: \mathrm{Tb}$ crystalline whiskers", Science and Technology of Advanced Materials, Vol.6, pp.215-218, Nov. 2004.

[4] Lionel Vayssieres, Karin Keis, Sten-Eric Lindquist, and Anders Hagfeldt, "Purpose-built anisotropic metal oxide material: 3D highly oriented microrod array of ZnO", Journal of Physical Chemistry B, Vol.105, pp.3350-3352, Mar. 2001.

[5] Xiangyang Ma, Hui Zhang, Yujie Ji, Jin Xu, and Deren Yang, "Sequential occurrence of $\mathrm{ZnO}$ nanopaticles, nanorods, and nanotips during hydrothermal process in a dilute aqueous solution", Materials Letters, Vol.59, pp.3393-3397, Jul. 2005.

[6] Jiangtao Hu, TeriI Wang Odom, and charles M. Lieber, "Chemistry and physics in one dimension: synthesis and properties of nanowires and nanotubes", Accounts of Chemical Research, Vol.32, No.5, pp.435445, Sep. 1998.

[7] Chun Cheng, Ming Lei, Lin Feng, Tai Lun Wong, K. M. Ho, Kwok Kwong Fung, Michael M. T. Loy, Dapeng $\mathrm{Yu}$, and Ning Wang, "High-quality $\mathrm{ZnO}$ nanowire arrays directly fabricated from photoresists", American Chemical Society Nano, Vol.3, No.1, pp.53-58, Dec. 2009.

[8] Jinhui Song, Jun Zhou, and Zhong Lin Wang, "Piezoelectric and semiconducting coupled power generating process of a single $\mathrm{ZnO}$ belt/wire. A technology for harvesting electricity from the environment", Nano Letters, Vol.6, No.8, pp.1656-1662, Jul. 2006.

[9] Min-Yeol Choi, Dukhyun Choi, Mi-Jin Jin, Insoo Kim, Sang-Hyeob Kim, Jae-Young Choi, Sang Yoon Lee, Jong Min Kim, and Sang-Woo Kim, "Mechanically powered transparent flexible charge-generating nanodevices with piezoelectric $\mathrm{ZnO}$ nanorods", $A d$ vanced Materials, Vol.21, pp.2185-2189, 2009.

[10] Jaejin Song and Sangwoo Lim, "Effect of seed layer on the growth of $\mathrm{ZnO}$ nanorods", The Journal of Physical Chemistry C, Vol.111, No.2, pp.596-600, Dec. 2006.

[11] Teng Ma, Min Guo, Mei Zhang, Yanjun Zhang, and Xidong Wang, "Density-controlled hydrothermal growth of well-aligned $\mathrm{ZnO}$ nanorod arrays", Nanotechnology, Vol.18, p.035605, Jan. 2007.

[12] Lori E. Greene, Matt Law, Joshua Goldberger, Frank- lin Kim, Justin C. Johnson, Yanfeng Zhang, Richard J. Saykally, and Peidong Yang, "Low-temperature wafer-scale production of $\mathrm{ZnO}$ nanowire arrays", Angewandte Chemie International Edition, Vol. 42, pp. 3031-3034, Mar. 2003.

[13] E. H. Rhoderick and R. H. Williams, Monographs in Electrical and Electronic Engineering (Oxford Science, Oxford), Vol.19, p.48, 1988.

[14] C. Q. Chen, Y. Shi, Y. S. Zhang, J. Zhu, and Y. J. Yan, "Size dependence of Young's modulus in $\mathrm{ZnO}$ nanowires", Physical Review Letters, Vol. 96, p.075505, Feb. 2006.

[15] Woong Lee, Min-Chang Jeong and Jae-Min Myoung, "Fabrication and application potential of $\mathrm{ZnO}$ nanowires grown on $\mathrm{GaAs}(002)$ substrates by metalorganic chemical vapour deposition", Nanotechnology, Vol.15, No.1, pp.254-259, Feb. 2004.

[16] Yifan Gao, and Zhong Lin Wang, "Equilibrium Potential of Free Charge Carriers in a Bent Piezoelectric Semiconductive Nanowire", Nano Letters, Vol. 9, pp.1103-1110, Mar. 2009.

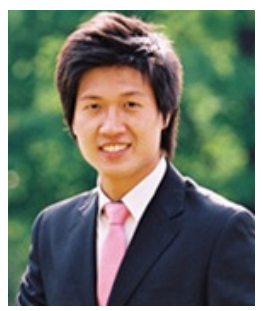

emitting diode.

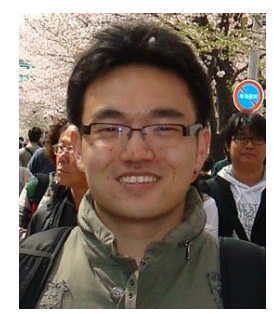

tions.

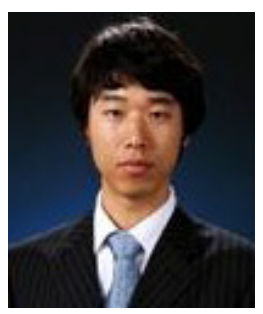

Chul Kim received BS degree in control and instrument engineering from Korea University. From 2010 he has studied for his MS degree at the Department of Micro/Nano systems at Korea University. His research interests are blood flow rate sensor and ANSYS simulation in MEMS and mi-

Jae-hoon Choi received BS degree in electrical engineering from Konkuk University. From 2009 he has studied for his MS degree at the School of Electrical Engineering at Korea University. His research interests are $\mathrm{ZnO}$ nano materials, superhydrophobic surface technology and $\mathrm{ZnO}$ based light

Xueqiu You received BS degree in electrical engineering in 2008 at Harbin institute of technology. He is currently an integrated $\mathrm{MS}$ and $\mathrm{PhD}$ student in the School of Electrical Engineering at Korea University. His research interests are $\mathrm{ZnO}$ and graphene nanostructures synthesis and applica- crofluidics. 


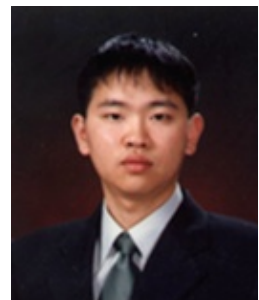

Jungil Park He received his BS degree in Division of Electronics and Information Engineering in 2004 at Korea University. $\mathrm{He}$ is currently an integrated MS and PhD student in the School of Electrical Engineering at Korea University. His research interests are microfabricated oxygen sensor and $\mathrm{ZnO}$ -

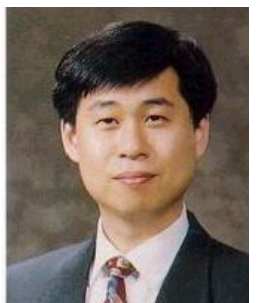

James Jungho Pak James Jungho Pak received his BS, MS and $\mathrm{PhD}$ degrees in Electrical Engineering respectively in 1985, 1988 and 1992 at Purdue University. He had worked in Intel Corporation in Santa Clara, CA, USA from 1992 to 1995 as a senior device physicist. Since 1995, he has been a professor in the School of Electrical Engineering at Korea University. His research interests include the Microsystems including bio-MEMS, biosensor, applications of polymer in microsensors and microactuators, flexible electronics and novel semiconductor devices and processing. 